\title{
CRINOIDEOS DEL GÉNERO Bactrocrinites DEL DEVÓNICO INFERIOR Y MEDIO DE LA CORDILLERA CANTÁBRICA (NO DE ESPAÑA)
}

\author{
Raimundo PIDAL \\ Departamento de Geología, Universidad de Oviedo, c/ Jesús Arias de Velasco, \\ s/n. 33005-Oviedo (España).rjpidal@gmail.com
}

Pidal, R. 2011. Crinoideos del género Bactrocrinites del Devónico Inferior y Medio de la Cordillera Cantábrica (NO de España). [Lower and Middle Devonian species of the genus Bactrocrinites (Crinoidea). Cantabrian Mountains (NW Spain).] Revista Española de Paleontología, 26 (1), 3-10. ISSN 0213-6937.

\begin{abstract}
This paper describes new forms of genus Bactrocrinites of both slopes of the Cantabrian Mountains. Thus in the asturian slope, three found specimens: Bactrocrinites longibrachiatus n. sp., Bactrocrinites aff. fusiformis and Bactrocrinites sp., they were gathered, respectively, in the Punta del Aguión, near Luanco, Aguión Formation (Upper Emsian); in the Cove of Moniello, Moniello Formation (Upper Emsian) and in the locality of Arbeyales, Santa Lucía Formation (Lower Eifelian). In the leonian slope: Bactrocrinites breimeri n. sp., Bactrocrinites truyolsi n. sp., a second form of Bactrocrinites aff. fusiformis and ? Bactrocrinites sp.; they were collected as well, first in Huergas de Gordón, Pola de Gordón, Portilla Formation (Givetian), the second also in the same locality, Santa Lucía Formation (Lower Eifelian), the third in Valporquero, Valle de Torío, La Vid Group (Upper Emsian) and the last in Colle, La Vid Group (Upper Emsian).

All the specimens gathered and described in the present work are relatively small and slender and show to remarkable affinities with the type species fusiformis.
\end{abstract}

Keywords: Crinoidea, Bactrocrinites, Lower and Middle Devonian, Cantabrian Mountains (NW Spain).

\section{RESUMEN}

Se describen nuevas formas de Bactrocrinites de ambas vertientes de la Cordillera Cantábrica. En la vertiente asturiana: Bactrocrinites longibrachiatus n. sp., Bactrocrinites aff. fusiformis y Bactrocrinites sp., proceden respectivamente de la Punta del Aguión, cerca de Luanco, Formación Aguión (Emsiense superior), de la Ensenada de Moniello, Luanco, base de la Formación Moniello (Emsiense superior) y de Arbeyales, Somiedo, techo de la Formación Santa Lucía (Eifeliense inferior). En la leonesa: Bactrocrinites breimeri n. sp., Bactrocrinites truyolsi n. sp., un segundo ejemplar de Bactrocrinites aff. fusiformis y ? Bactrocrinites sp.; los dos primeros proceden de Huergas de Gordón, Pola de Gordón, en la Formación Portilla (Givetiense) y Formación Santa Lucía (Eifeliense inferior), respectivamente, el tercero, de Valporquero, Valle de Torío, Grupo La Vid (Emsiense superior) y el último de Colle, también en el Grupo La Vid (Emsiense superior).

Todas las especies recogidas y descritas en el presente trabajo son relativamente pequeñas y esbeltas y muestran evidentes afinidades con la especie tipo fusiformis.

Palabras clave: Crinoideos, Bactrocrinites, Devónico Inferior y Medio, Cordillera Cantábrica (NO de España).

\section{INTRODUCCIÓN}

La primera referencia sobre los bactrocrinítidos españoles figura en una monografía publicada en el año 1962 por el Profesor A. Breimer. En ella se cita la existencia del espécimen TB73, Bactrocrinites spec. (sic), recogido en las inmediaciones de la localidad leonesa de Colle (Fig.
1), en el Grupo La Vid (Emsiense), "Nivel Crin 1" (probablemente equivalente al "Nivel 27" de García-Alcalde, 1996), depositado en la colección del Instituto Geológico y Minero de España (IGME) de Madrid.

Décadas más tarde, Hauser \& Landeta (2007), señalan la existencia de un nuevo ejemplar procedente de Mirantes de Luna, León: Bactrocrinites aff. depresuss Schult- 
ze, 1866. Pidal (2008) encontró en la misma localidad de Colle, y posiblemente en el mismo nivel, nuevo material del género que asignó a una nueva especie: Bactrocrinites robustus, y aproximó la forma de Breimer a Bactrocrinites muelleri Jaekel, 1895.

Nuevas campañas de prospección nos permitieron descubrir nuevo material en la vertiente leonesa: Bactrocrinites breimeri n. sp., Bactrocrinites truyolsi n. sp., Bactrocrinites aff. fusiformis (Roemer, 1844) y ? Bactrocrinites sp. y ampliar el ámbito de distribución geográfica del género a la vertiente asturiana de la Cordillera Cantábrica con Bactrocrinites longibrachiatus n. sp., Bactrocrinites aff. fusiformis y Bactrocrinites sp. Estos hallazgos amplían igualmente la distribución estratigráfica del género en la Cordillera Cantábrica, desde el Emsiense al Givetiense.

Desde el punto de vista paleoecológico, estas especies bentónicas, vivieron en un entorno nerítico y somero en consonancia con las rocas en las que fueron encontradas (García-Alcalde et al., 1979, 2002; Álvarez \& Brime, 1982; Méndez-Bedia \& Soto, 1984; Álvarez, 1990; García-Alcalde, 1996, 1998).

\section{SISTEMÁTICA}

Todo el material aquí referenciado está depositado en la colección del Museo de Geología (Área de Paleontología) de la Universidad de Oviedo (España).

Clase CRINOIDEA Miller, 1821

Subclase INADUNATA Moore \& Laudon, 1943

Orden CLADIDA Moore \& Laudon, 1943

Familia Bactrocrinitidae Jaekel, 1918

Género Bactrocrinites Schnur, 1849

Especie-tipo: Poteriocrinus fusiformis Roemer, 1844

\section{Bactrocrinites longibrachiatus n. sp. Figs. 4a, 5a-5b, Tabla 1}

Derivatio Nominis: hace alusión a la longitud de sus brazos.

Material: un único ejemplar, el Holotipo DPO 113603 en estado aceptable, conservando la región posterior del cáliz, la parte superior de la probóscide anal, algunos brazos y el primer tramo del tallo.

Localidad y estrato típicos: fue recogido en la Punta del Aguión (Fig. 2), pequeño cabo al NO de la villa de Luanco (Gozón-Asturias), en la Formación Aguión, en la parte superior del Grupo Rañeces, próximo al límite con la Formación Moniello (Emsiense superior).

Diagnosis: cáliz pequeño, de perfil longitudinal cónico y base moderadamente ancha, con placas convexas de relieve suave; basales aparentemente equidimensionales; radiales ligeramente mayores que el resto; anal X desarrollada, de tamaño similar a las radiales y en línea con ellas; probóscide anal larga rematada por un penacho de placas espinosas; brazos largos y gruesos en el primer tramo; tallo heteromorfo de sección pentagonal estrellada. Ornamentación granular débil.

Calyx small, conical longitudinal profile and base moderately wide, with convex and smooth relief plates; $b a-$ sals apparently equal in size; radials slightly bigger than other plates; anal $X$ developed, similar in size to the radials and in line with them; anal sac long with spinose plates at summit; long arms and stout in the first section: stem heteromorphic pentagonal stellate in section; weak granular ornamentation.

Descripción: cáliz de pequeño tamaño y perfil longitudinal cónico aplanado en la base, formado por placas convexas de escaso relieve y ornamentación suave.

Círculo infrabasal constituido por cinco placas pentagonales, más anchas que altas.

Cinco basales equidimensionales, tres pentagonales y dos hexagonales, la posterior y la posterior derecha.

Cinco radiales pentagonales, más anchas que altas.

\section{MAR CANTÁBRICO}

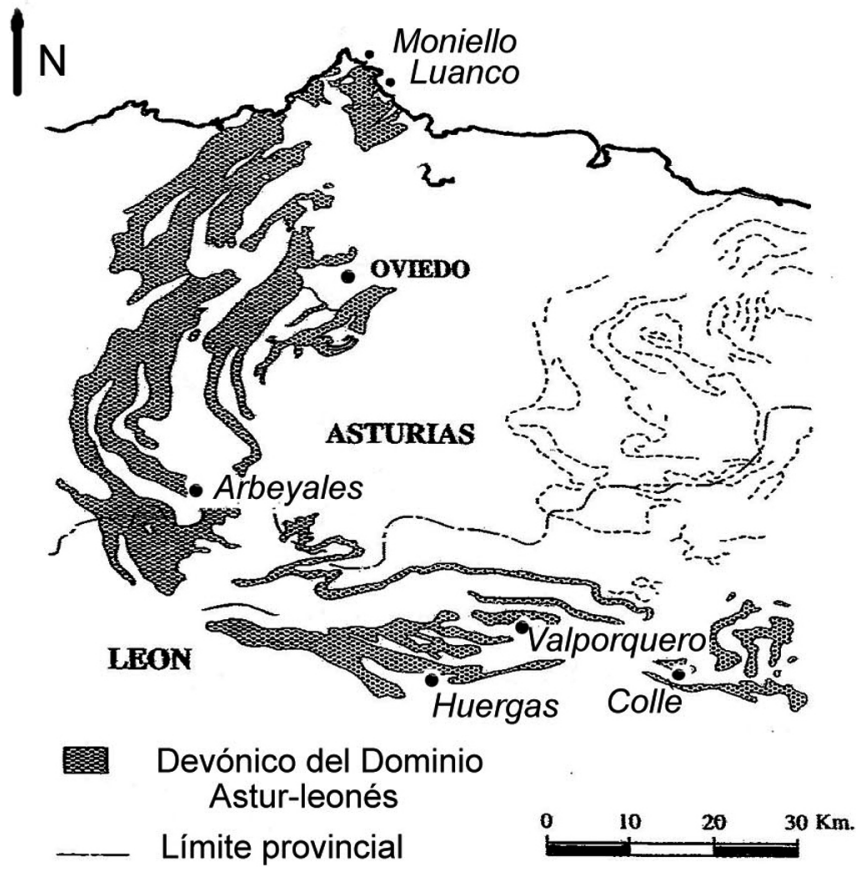

Figura 1. Situación geográfica y estratigráfica de las localidades en las que fueron hallados los distintos especímenes del género Bactrocrinites (modificado de García-Alcalde, 1996).

Geographical and stratigraphical situation of the localities where different specimens of the genus Bactrocrinites were found (modified from Garcia-Alcalde, 1996). 
Tabla 1. Medidas de los distintos especímenes de Bactrocrinites expresadas en mm. Th/Mw, altura total/ancho máximo y, MVA y MTA, ejes medio vertical y transversal máximo, respectivamente.

Measurements (in $\mathrm{mm}$ ) of the different specimens of Bactrocrinites. Th/Mw, total height/maximum width and MVA and $M T A$, mean vertical axis and mean maximum transversal axis, respectively.

\begin{tabular}{|c|c|c|c|c|c|c|c|c|c|c|c|}
\hline $\begin{array}{l}\text { Espécimen } \\
\text { DPO: }\end{array}$ & Altura total & $\begin{array}{l}\text { Anchura } \\
\text { máxima }\end{array}$ & $\begin{array}{c}\text { Índice } \mathrm{Th} / \\
\mathrm{Mw}\end{array}$ & $\begin{array}{c}\text { IIB MVA } \\
\text { MTA }\end{array}$ & & $\begin{array}{c}\text { BB MVA } \\
\text { MTA }\end{array}$ & & $\begin{array}{r}\text { RR MV } \\
\text { MTA }\end{array}$ & & Radianal & Anal X \\
\hline 113603 & 10 & 11 & 0,91 & 3 & 4 & 4 & 4 & 4 & 4,6 & 3 & 4 \\
\hline 113600 & & 17 & & & & 12,5 & 8 & 6 & 8 & 4 & 4 \\
\hline 113604 & 14 & 11 & 1,27 & 4,5 & 3,6 & 4,8 & 3,6 & 3,6 & 4,5 & & \\
\hline 113596 & 13 & 9,5 & 1,37 & 4 & 3 & 7 & 5 & 3 & 4,5 & 2 & 2 \\
\hline 113597 & 13 & 9,5 & 1,37 & 4 & 3 & 6 & 5 & 3 & 4,5 & 2 & 2 \\
\hline 113598 & 26 & 17 & 1,53 & 9 & 6 & 12 & 8 & $5(6)$ & $8(9)$ & 4 & 56 \\
\hline 113599 & & 14 & & & & 10 & 7 & 5 & 6,5 & 4 & 5 \\
\hline 113605 & 6 & 6 & 1 & 2 & 2 & 3 & & 2 & 2 & & \\
\hline
\end{tabular}

\section{MAR CANTÁBRICO}

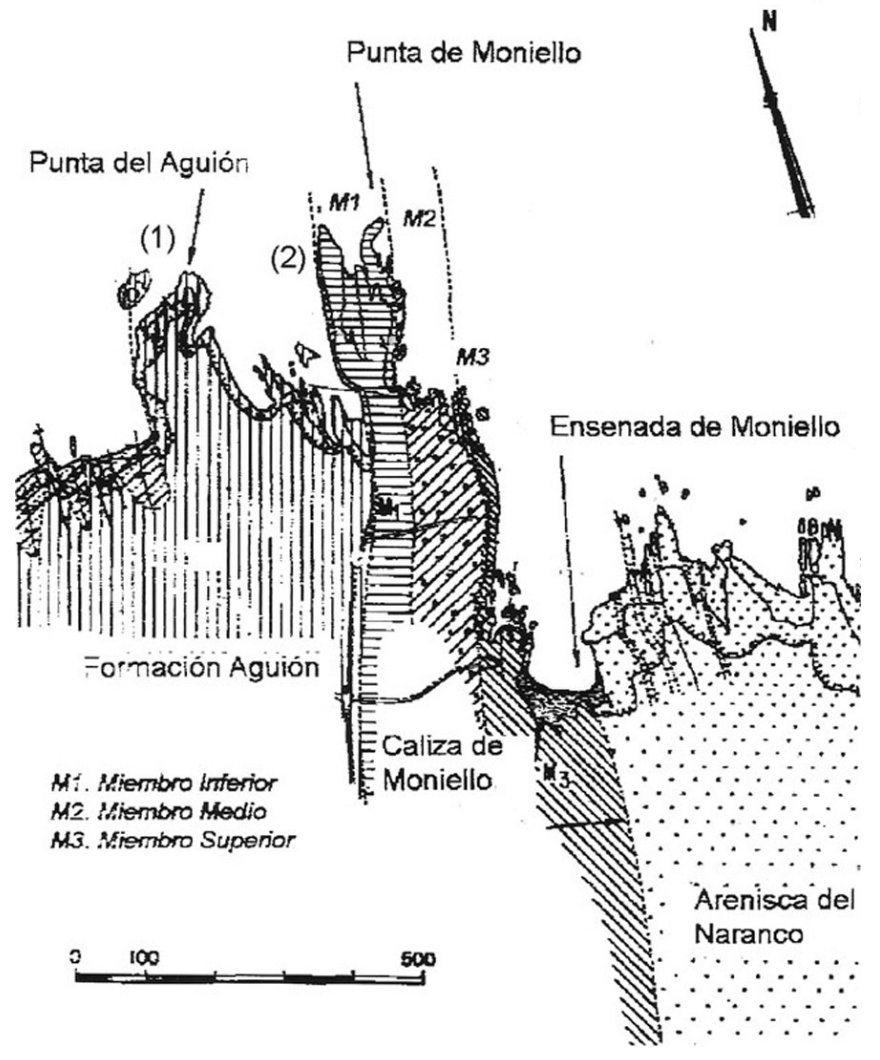

Figura 2. Situación geográfica y estratigráfica de los yacimientos correspondientes a: (1) Bactrocrinites longibrachiatus n. sp. y (2) Bactrocrinites aff. fusiformis (Roemer, 1844) (modificado de Truyols \& Julivert, 1976). Geographical and stratigraphical situation of occurrences of: (1) Bactrocrinites longibrachiatus n. sp. and (2) Bactrocrinites aff. fusiformis (Roemer, 1844) (modified from Truyols \& Julivert, 1976).
Radianal y la anal X en la copa; la primera, subcuadrangular, se dispone sobre las basales posterior y posterior derecha, adoralmente está en contacto con la anal $\mathrm{X}$ y la radial posterior derecha (radial C); la segunda, pentagonal, en línea con las radiales y casi del mismo tamaño que éstas, se sitúa sobre la radianal y la basal posterior.

El tegmen, mal conservado, formado por numerosas placas de aspecto frágil, está rematado por un penacho de placas espinosas más grandes que el resto. El aparato braquial muestra unos brazos largos e isotomos; la serie de primibraquiales está formada por tres placas gruesas respecto del resto, las dos primeras son subrectangulares y la tercera, subtriangular, siendo más alta que las anteriores; las secundibraquiales, 13 o 14 y las tertibraquiales, más finas, en número superior a 20. Cabe señalar que la numeración y descripción de una parte de las braquiales es difícil debido a su mal estado.

El tallo, heteromorfo, de sección pentagonal estrellada, es grueso; sólo se conserva un tramo proximal de $7 \mathrm{~mm}$ con signos evidentes de disolución; las tres primeras placas, aparentemente iguales en grosor, muestran un diámetro sensiblemente más ancho que el resto; la noditaxis (nudo-entrenudo) del conjunto de piezas columnares restante podría ajustarse a la secuencia siguiente: $\mathrm{N}, 2 \mathrm{IN}, 3 \mathrm{IN}, 2 \mathrm{IN}, 3 \mathrm{IN}, 2 \mathrm{IN}$.

La observación cuidadosa de las placas permite identificar en algunos puntos de las mismas indicios de ornamentación granular.

Discusión: la inclusión de $B$. longibrachiatus $\mathrm{n}$. sp. en el género Bactrocrinites se justifica por la existencia de afinidades morfológicas con la especie tipo B. fusiformis (Roemer, 1844) como son el perfil de cáliz, número, forma y disposición general de las placas tecales, etc.

Nuestra especie se diferencia, en general, de todas las formas conocidas del género por presentar un cáliz menos esbelto, círculo infrabasal con placas más anchas que altas, basales prácticamente equidimensionales, radiales ligeramente más anchas que altas y anal $\mathrm{X}$ proporcionalmente más desarrollada. De B. robustus, además, en el perfil 
cónico del cáliz y, de B. penaneachensis Le Menn, 1985, en que las series de la copa no son equidimensionales.

\section{Bactrocrinites aff. fusiformis (Roemer, 1844)}

Figs. 4b-4c, 5c-5d, Tabla 1

Material y procedencia: dos ejemplares, DPO 113599 y 113600 , conservándose en relativo buen estado una porción del cáliz; infrabasales, brazos y tallo, ausentes. Procedente, el primero, de Valporquero (Fig. 1), valle de Torío (León), de unas margas calcáreas y pizarras situadas en la parte alta del Grupo La Vid, yacimiento VT. LV-6 (Emsiense superior) y, el segundo, de la Ensenada de Moniello (Fig. 2), Luanco (Asturias), de las calizas de la base de la Formación Moniello, nivel M-804 (Emsiense superior).

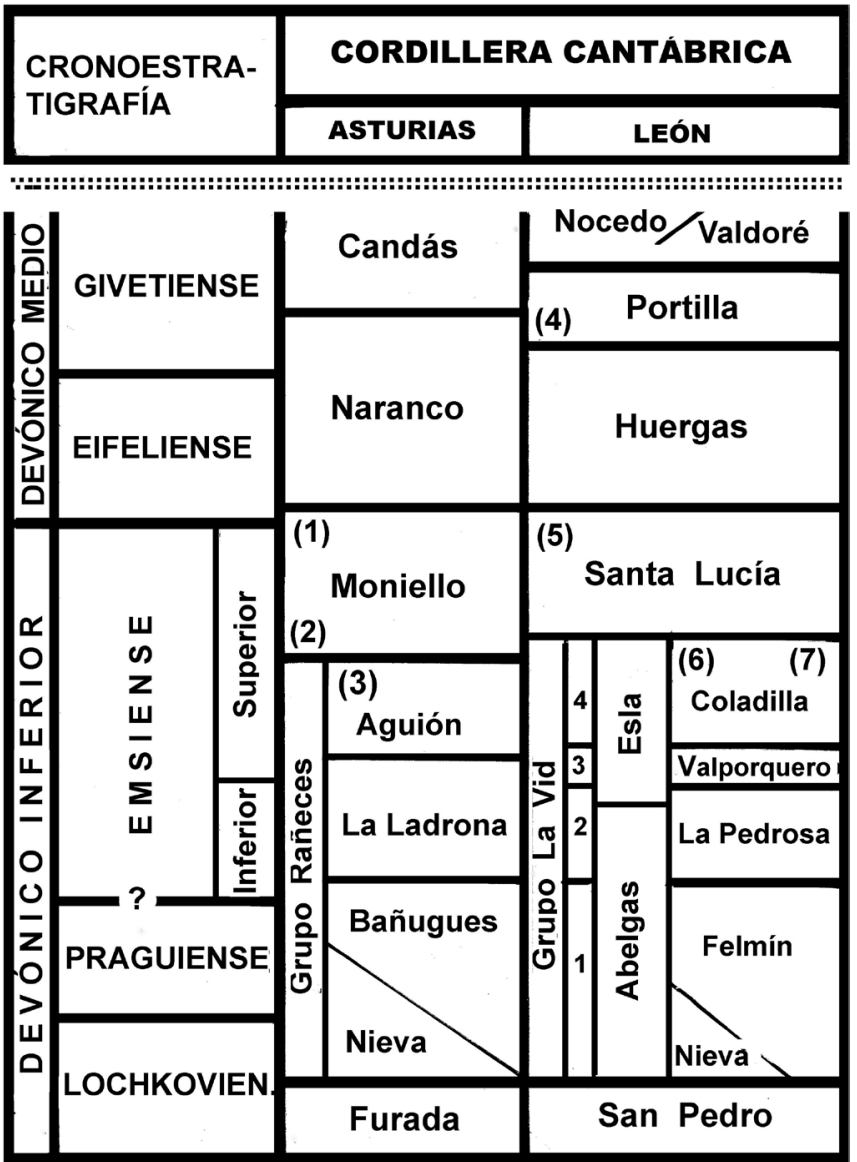

Figura 3. Columna cronoestratigráfica del Devónico Astur-leonés en la que se indica, mediante números, la posición aproximada de los yacimientos donde se hallaron los diferentes ejemplares del género Bactrocrinites: (1) Bactrocrinites sp.; (2) B. aff. fusiformis; (3) B. longibrachiatus n. sp.; (4) B. breimeri n. sp.; (5) B. truyolsi n. sp.; (6) B. aff. fusiformis; (7) ? Bactrocrinites sp. Chronoestratigraphical column of the Devonian in the Asturo-leonese domain. Numbers indicate approximate positions of Bactrocrinites occurrences: (1) Bactrocrinites sp.; (2) B. aff. fusiformis; (3) B. longibrachiatus $n$. sp.; (4) B. breimeri n. sp.; (5) B. truyolsi n. $s p$.; (6) B. aff. fusiformis; (7) ? Bactrocrinites $s p$.
Discusión: la aproximación de estos dos ejemplares incompletos a la especie tipo B. fusiformis se justifica por presentar una copa alta y cónica con placas de apreciable espesor; basales más altas que anchas; radiales más anchas que altas (ocasionalmente en fusiformis); radianal y anal $\mathrm{X}$ en la copa, cuadrangular la primera y pentagonal la segunda, etc.

En el caso del DPO 113600, la diferencia más significativa respecto de la especie tipo radica en la anal $\mathrm{X}$, ya que se sitúa apreciablemente por debajo de la línea superior de las radiales y es de un tamaño significativamente menor que éstas.

\section{Bactrocrinites sp.}

Fig. 5e-5f, Tabla 1

Material y procedencia: un único ejemplar, el DPO 113604 del que, en la actualidad, sólo se conserva una porción del ta1lo, ya que el cáliz fue extraviado durante un traslado. Afortunadamente se dispone de la descripción del mismo así como de información gráfica precisa. Dicho cáliz presenta signos de deformación por compresión lateral y de disolución en algunas zonas. En mal estado, las radiales posteriores, área anal y tegmen. Brazos, ausentes.

Fue recogido en el techo de la Formación Santa Lucía (Eifeliense inferior), en el primer nivel pizarroso-calcáreo próximo a la Caliza Masiva de la localidad asturiana de Arbeyales (Fig. 1), Somiedo (Asturias).

Descripción: crinoideo de talla pequeña, que muestra un perfil longitudinal cónico-alargado aplanado en la base.

El círculo infrabasal está formado por cinco piezas pentagonales, más altas que anchas, ligeramente más anchas adoralmente; los lados superiores muestran una apreciable concavidad.

El círculo basal está constituido por cinco piezas hexagonales, más altas que anchas y de tamaño y forma similar al de las infrabasales.

El círculo radial está formado a su vez por cinco piezas pentagonales características, más anchas que altas, que se ensanchan a su vez en la base; la foseta de inserción de los brazos presenta una suave pendiente o inclinación en sentido aboral.

La presencia de una anal X en la copa es segura ya que se puede identificar un pequeño fragmento de la misma en posición interradial a nivel de las fosetas de inserción de los brazos. El mal estado del área anal no nos permite ir más allá. El tegmen, plano, está muy deteriorado; se pueden identificar en él, no obstante, un número reducido de placas.

Los brazos, ausentes, serían proporcionalmente gruesos en el inicio dadas las características de las fosetas de inserción de los mismos, dichas fosetas ocupan prácticamente todo el área superior.

El tallo, grueso y heteromórfico, presenta una sección transversal pentalobada; se conservan dos fragmentos del mismo, uno de ellos proximal, ambos con similar organización. La noditaxis (nudo-entrenudo) muestra la siguiente secuencia: N, 3IN, 2IN, 3IN, 1IN, 3IN, 2IN, 3IN; los nudos destacan sobre las restantes piezas columnares, siendo su espesor de $1 \mathrm{~mm}$.

El segundo fragmento termina en una placa $3 \mathrm{IN}$ en la que se puede observar lo siguiente: una aureola formada por 5 lóbulos 
petaloideos deprimidos, áreas interpetaloideas en las que destacan sendas crestas prominentes cuya altura decrece progresivamente hacia el exterior y una suave crenulación que se inicia en el mismo borde externo de la placa; el lumen no se conserva. No se aprecia ornamentación.

Discusión: la inclusión de este ejemplar del Devónico asturiano en el género Bactrocrinites se justifica por presentar afinidades con la especie tipo fusiformis como el perfil del cáliz, número, forma y disposición de las placas tecales.

Nuestra especie se diferencia en general de todas las formas conocidas del género por presentar la sección longitudinal del cáliz distinta, en copa abierta, en la que las radiales características se expanden lateralmente. De $B$. robustus, además, en el perfil cónico del cáliz.

Por último, señalar que esta forma pudiera ser considerada como una especie nueva, pero las condiciones que la rodean: extravío y deficiente conservación, aconsejan por prudencia clasificarla como Bactrocrinites sp..

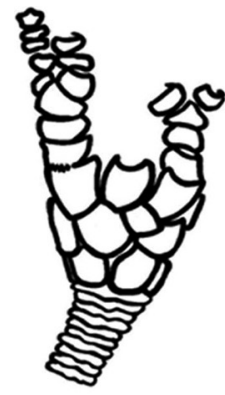

$10 \mathrm{~mm}$

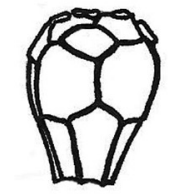

$10 \mathrm{~mm}$

d
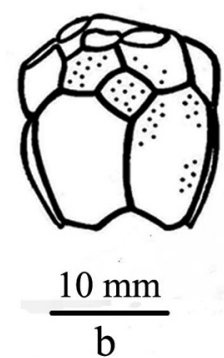

b

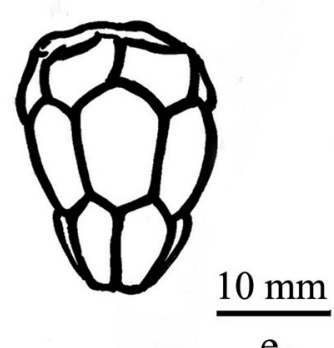

e

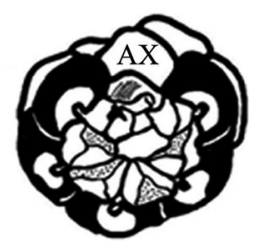

$10 \mathrm{~mm}$

C

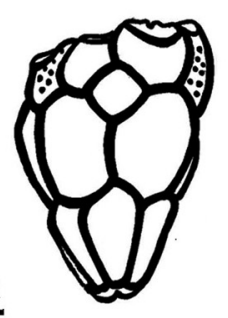

\section{Bactrocrinites breimeri n. sp. Figs. 4d, 5g, Tabla 1}

Derivatio nominis: dedicado al Profesor D. Albert Breimer de la Universidad de Leiden, que describió por primera vez un bactrocrino de la Cordillera Cantábrica.

Material: dos ejemplares de similar aspecto y dimensiones, el Holotipo DPO 113596 y el Paratipo DPO 113597. Brazos y ta-

Localidad y estrato típicos: ambos especímenes fueron recogidos en un tramo margo-pizarroso situado en la base de las calizas de la Formación Portilla (Givetiense), aflorantes en la sección Huergas-Gordón (Fig. 1), Pola de Gordón (León).

Diagnosis: cáliz de reducidas dimensiones, de perfil longitudinal cónico-globoso característico formado por placas con escaso relieve; radiales combándose adoralmente a partir del tercio superior; radianal y anal X convergentes adoralmente, éstas dos últimas prácticamente iguales; tegmen constituido por un número reducido de placas, probóscide anal poco desarrollada; fosetas de inserción de los brazos con escasa y fuerte crenulación.

Calyx small, conical-globular longitudinal profile characteristic made up of smooth relief plates; upper third of radials bending adoraly as of upper third; radianal and anal $X$ adoral converging, those two last practically equals; tegmen formed by a reduced number of plates, anal sac weakly developed; facets for the insertion of the arms with scarce and thick crenulation.

Descripción: crinoideo de talla pequeña, dicíclico, que muestra un perfil longitudinal cónico-globoso característico constituido por placas de suave relieve.

El círculo infrabasal está constituido por cinco placas pentagonales más altas que anchas.

Cinco basales, más altas que anchas, tres pentagonales y dos hexagonales, la posterior y la posterior derecha, siendo estas últimas ligeramente más anchas que el resto; cinco radiales pentagonales ligeramente convexas, más anchas que altas, algo menores las que están en contacto con las anales.

La radianal y la anal X constituyen el área anal; la primera, subcuadrangular, limita aboralmente con las basales posterior y posterior derecha y, adoralmente, con la anal X y la radial posterior derecha; la anal X, algo mayor, es pentagonal, se sustenta aboralmente sobre la radianal y la basal posterior; en continuidad con su lado superior, ya en el tegmen, se disponen las piezas de la probóscide.

A nivel del tercio superior del círculo basal, el cáliz empieza a combarse adoralmente, reduciendo sensiblemente su anchura en el límite superior de las radiales, característica ésta de breimeri.

Las fosetas en las que se insertan los brazos son anchas y con un ribete que resalta su contorno externo; muestran una crenulación escasa y fuerte.

El tegmen, plano, está constituido por un número reducido de placas, destacando del resto, en tamaño y forma, cuatro piezas sagitadas situadas en posición interambulacral. En continuidad con la anal $\mathrm{X}$ se puede observar una probóscide anal de 1lo, ausentes.
B. breimeri $n$. sp. (posterior view); e, DPO 113598, B truyolsi $n$. sp. (anterior and posterior view respectively). 


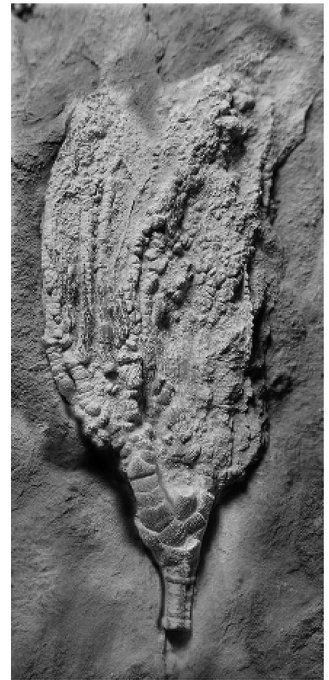

a

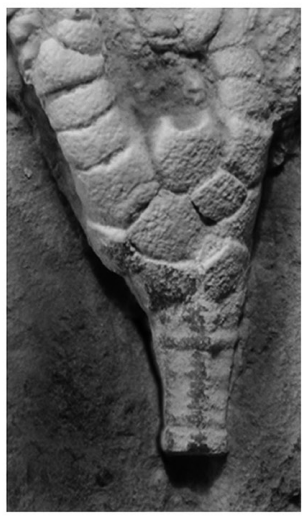

b

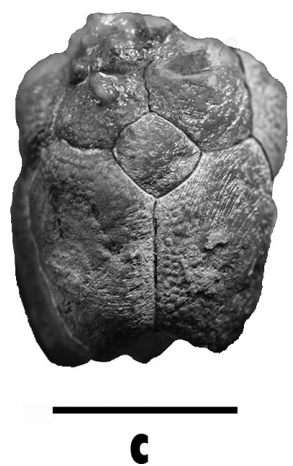

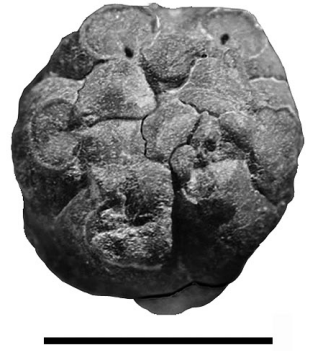

d

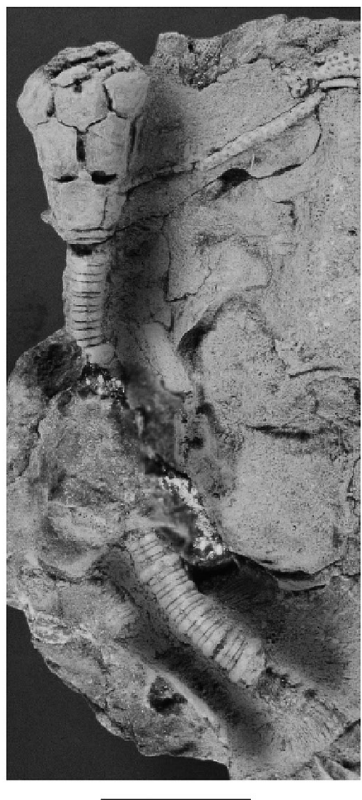

e

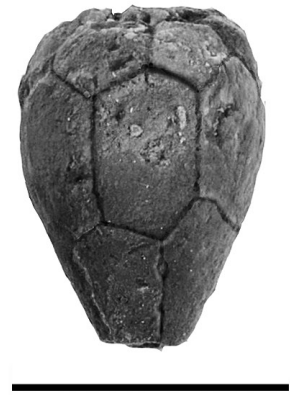

g
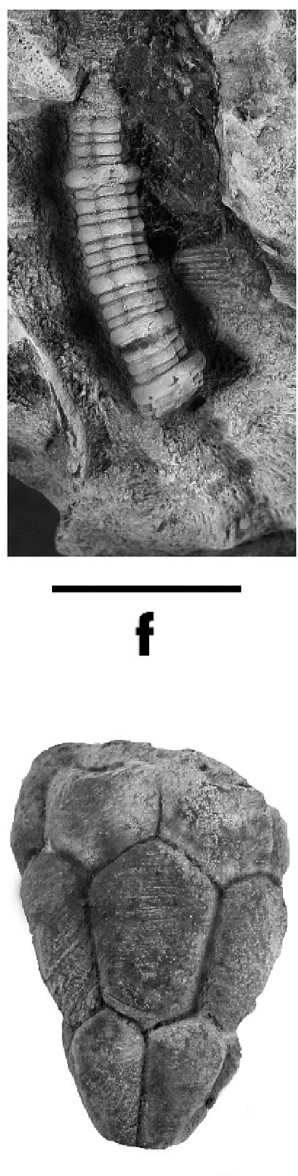

h
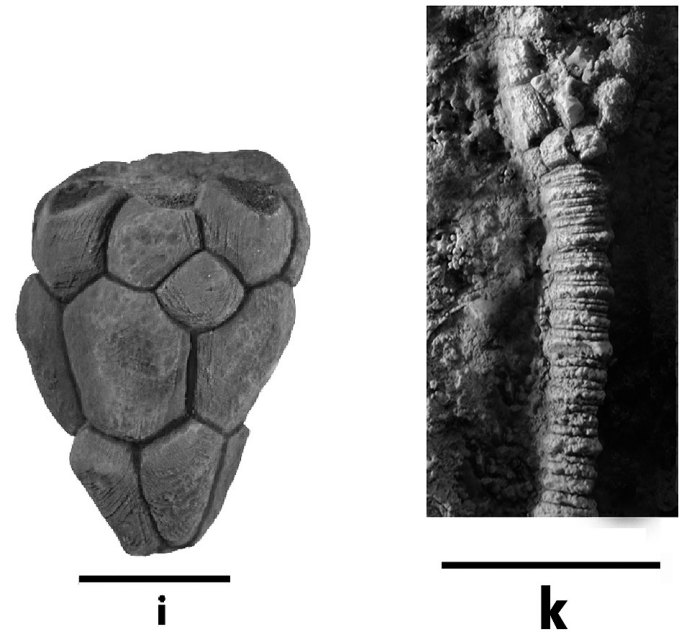

Figura 5. Bactrocrinites longibrachiatus n. sp., DPO 113603, a-b: vista general y detalle del cáliz respectivamente. Bactrocrinites aff. fusiformis (Roemer, 1844), DPO 113599 y 113600 , c-d: vistas de la porción del cáliz y del área oral respectivamente. Bactrocrinites sp., DPO 113604, e-f: vistas general y de un fragmento del tallo respectivamente. Bactrocrinites breimeri n. sp., DPO 113596, g: vista general del cáliz. Bactrocrinites truyolsi n. sp., DPO 113598, h-i: áreas anterior y posterior respectivamente. ?Bactrocrinites sp., DPO 113605, j-k: vista general y detalle del cáliz y tallo respectivamente. Todos los ejemplares blanqueados con óxido de magnesio. Escala gráfica $=10 \mathrm{~mm}$.

Bactrocrinites longibrachiatus $n$. sp., DPO 113603, a-b: general view and detail of the calyx respectively. Bactrocrinites aff. fusiformis (Roemer, 1844), DPO 113599 and 113600, c-d: incomplete calyx and oral views respectively. Bactrocrinites sp., DPO 113604, e-f: general and stem fragment views respectively. Bactrocrinites breimeri $n$. sp., DPO 113596, g: general view of the calyx. Bactrocrinites truyolsi $n$. sp., DPO 113598, $\boldsymbol{h}$-i: anterior and posterior views respectively. ?Bactrocrinites sp. DPO 113605, $\boldsymbol{j}$-k: general view and detail of the calyx and stem respectively. All specimens whitened whith magnesium oxide. Bar scale $=10 \mathrm{~mm}$. 
reducidas dimensiones en posición subcentral, dicha probóscide está flanqueada lateralmente por dos piezas seudohexagonales y, frontalmente, por una rómbica cuya diagonal mayor se dispone normalmente a la misma.

No se observa ornamentación.

Discusión: la inclusión de B. breimeri n. sp. en el género Bactrocrinites se justifica por las afinidades morfológicas con la especie tipo B. fusiformis como son el perfil de la copa dorsal alto y cónico, número, forma y disposición de las placas tecales, etc. De todas las formas conocidas del género se diferencia, en general, por presentar un cáliz globoso característico.

\section{Bactrocrinites truyolsi n. sp. Figs. 4e, 5h-5i, Tabla 1}

Derivatio nominis: dedicado al Profesor D. Jaime Truyols, de la Universidad de Oviedo, a quien tanto debo en lo personal y en lo académico.

Material: el Holotipo DPO 113598, del que se conserva el cáliz en relativo buen estado con una ligera compresión lateral. Tegmen, brazos y tallo, ausentes.

Localidad y estrato típicos: recogido en el techo de la Formación Santa Lucía (Eifeliense), en la sección Huergas-Gordón (Fig. 1), Pola de Gordón (León).

Diagnosis: cáliz de tamaño medio, de sección longitudinal cónica, constituido por placas de acusado relieve; basales significativamente más altas que anchas y mayores que el resto; radiales apreciablemente más anchas que altas; ornamentación granular.

Calyx medium, conical in longitudinal section, formed by plates of thick relief; basals significant higher than wide and bigger than other plates; radials appreciably wider than high; granular ornamentation.

Descripción: el cáliz, de tamaño medio, dicíclico, de perfil longitudinal cónico, constituido por placas gruesas características. El círculo infrabasal está constituido por cinco placas pentagonales, más altas que anchas.

Cinco basales más altas que anchas, tres hexagonales y, heptagonales, la posterior y la posterior derecha. Las radiales, cinco, pentagonales, de menor tamaño las que están en contacto con la anal X, significativamente más anchas que altas; las fosetas de inserción de los brazos presentan una suave inclinación aboral. En la copa se encuentran la radianal y la anal X, subcuadrangular y pentagonal respectivamente; la primera en contacto aboral con las basales posterior y posterior derecha $\mathrm{y}$, adoralmente con la anal X y la radial posterior derecha, a su vez, la anal X, en línea con las radiales, se sustenta sobre la radianal y la basal posterior. Tegmen, brazos y tallo no se conservan. Presenta ornamentación granular.

Discusión: la inclusión de $B$. truyolsi n. sp. en el género Bactrocrinites se justifica por presentar afinidades morfológicas con la especie tipo B. fusiformis, como son el perfil longitudinal del cáliz, número, forma y distribución de las placas tecales.

De todas las especies que conocemos de Bactrocrinites se diferencia, en general, por poseer un cáliz menos esbelto y el acusado relieve de las placas que lo forman. De $B$. robustus, en que su cáliz es más masivo, las placas que lo forman recuerdan a las de $B$. truyolsi, aunque son menos esbeltas que las de éste. De algunos ejemplares de B. muelleri (ver Bohatý, 2005), en el perfil del cáliz, apreciablemente más cónico en nuestra especie.

\section{? Bactrocrinites sp. Fig. 5j-5k, Tabla}

Material y procedencia: un único ejemplar, el DPO 113605 del que se conserva el cáliz incompleto, brazos y un primer tramo del tallo, todos con evidentes signos de disolución. Fue recogido en el Grupo La Vid, yacimiento I-54 (Emsiense superior), a unos $15 \mathrm{~m}$ por debajo de la Formación Santa Lucía, en la localidad leonesa de Colle (Fig. 1).

Descripción: crinoideo de tamaño pequeño, de perfil cónico aplanado en la base, constituido por piezas de apreciable espesor, infrabasales y radiales iguales.

El círculo infrabasal está formado por placas pentagonales equidimensionales anchas en la base. El círculo basal está constituido por piezas hexagonales aparentemente equidimensionales y de mayor tamaño que las infrabasales y las radiales. Las radiales son pentagonales. El estado general del cáliz no permite establecer mayores precisiones.

El aparato braquial muestra unos brazos largos e isotomos; las series primibraquiales están formadas por cuatro placas gruesas respecto del resto; a partir de las secundibraquiales las placas se van adelgazando progresivamente. El mal estado del conjunto no permite una descripción más detallada.

El tallo, heteromórfico y de sección transversal subpentagonal, es grueso; se conserva un tramo proximal de unos 20 $\mathrm{mm}$ con signos evidentes de disolución; su diámetro medio es de unos $3,5 \mathrm{~mm}$; las primeras placas tienen un diámetro ligeramente superior al resto; la noditaxis (nudo-entrenudo) muestra la siguiente frecuencia: N 2IN 1IN 2IN, las placas se engrosan distalmente de forma progresiva.

Discusión: la existencia de afinidades morfológicas entre este ejemplar del Devónico asturiano y la especie tipo $B$. fusiformis como son el perfil del cáliz, forma y disposición de las placas, etc., permitirían clasificar nuestro taxón como Bactrocrinites sp. No obstante, una actuación rigurosa en ese sentido, teniendo en cuenta su estado de conservación, aconsejan clasificarlo como ? Bactrocrinites sp. De las formas conocidas, nuestra especie se diferencia en general por presentar las series de infrabasales y radiales equidimensionales.

\section{AGRADECIMIENTOS}

Deseo mostrar mi especial agradecimiento a los Profesores revisores D. Jenaro García-Alcalde del Departamento de Geo- 
logía de la Universidad de Oviedo por animar, dirigir, y orientar el presente trabajo, a D. Jean Le Menn de la Universidad de la Bretaña Occidental cuyas opiniones sobre el campo de los crinoideos son tenidas por mí en muy alta estima, a D. Miguel V. Pardo Alonso de la Universitat de València por su comprensión, meticulosidad y paciencia, así como a todas las personas que me brindaron en su momento apoyo técnico, caso de D. Joaquín Vázquez, fotógrafo del Departamento.

\section{BIBLIOGRAFÍA}

Álvarez, F. 1990. Devonian Athyrid brachiopods from the Cantabrian Zone (NW Spain). Biostratigraphie du Palézoïque, 11, 1-311.

Álvarez, F. \& Brime, C. 1982. Aportaciones al conocimiento de las condiciones de formación de algunos depósitos fosilíferos del Devónico Cantábrico. Trabajos de Geología, Universidad de Oviedo, 12, 153-157.

Bohatý, J. 2005. Bactrocrinites (Crinoidea) aus dem Mitteldevon der Eifel (linksrheinisches Schiefergebirge, Deutschland) - Taxonomie, Biostratigraphie und Fazieskontrolle. Neues Jahrbuch fur Geologie und Paläontologie, Abhandlungen, 235, 381-410.

Breimer, A. 1962. A monograph on Spanish Palaeozoic Crinoidea. Leidse Geologische Mededelingen, 27, 1-190.

García-Alcalde, J. L. 1996. El Devónico del dominio AsturLeonés en la Zona Cantábrica ( $\mathrm{N}$ de España). Revista Española de Paleontología, $\mathbf{n}^{\mathbf{0}}$ extra., 78-51.

García-Alcalde, J. L. 1998. Terebratúlidos (Braquiópodos) del Emsiense superior de Colle (Sabero, León, N. de España). Trabajos de Geología, Universidad de Oviedo, 21, 159-176.

García-Alcalde, J. L., Arbizu, M. A., García-López, S. \& Méndez-Bedia, I. 1979. Meeting of the International Subcomission on Devonian Stratigraphy - Guidebook of the Field Trip. Servicio de Publicaciones de la Universidad de Oviedo, 42 pp.

García-Alcalde, J. L., Carls, P., Pardo Alonso, M. V., Sanz López, J., Soto, F., Truyols-Massoni, M. \& ValenzuelaRíos, J. I. 2002. Devonian. In: The Geology of Spain (eds. W. Gibbons \& T. Moreno). The Geological Society of London, 67-91.
Hauser, J. \& Gómez Landeta, F. 2007. Neue Crinoiden aus dem Paläozoikum von Nordspanien mit einem Beitrag zu Lepidocentrus aus dem mittleren Emsium. Eigenverlag, Bonn, $78 \mathrm{pp}$.

Jaekel, O. 1895. Beiträge zur Kenntnis der palaeozoischen Crinoiden Deutschlands. Paläontologisches Abhandlungen, 7, 1-116.

Jaekel, O. 1918. Phylogenie und System der Pelmatozoen. Paläontologische Zeitschrift, 3, 1-128.

Le Menn, J. 1985. Les Crinoïds du Dévonien Inferieur et Moyen du Massif Armoricaine. Mémoires de la Société Géologique et Minéralogique de Bretagne, 30, 268 pp.

Méndez-Bedia, I. \& Soto, F. 1984. Paleoecological succession in a Devonian organic buildup (Moniello Fm., Cantabrian Mountains, NW Spain). Geobios, 8, 151-157.

Miller, J. S. 1821. A natural History of the crinoidea or Lily-shaped Animals, whith observations of the Genera Asteria, Euryale, Comatula and Marsupites. Bryan \& Co., Bristol, 150 pp.

Moore, R. C. \& Laudon, L. R. 1943. Evolution and classification of Paleozoic crinoids. Geological Society of America, Special Paper, 46, 153 pp.

Pidal, R. 2008. A Lower Devonian (Emsian) Species of the Genus Bactrocrinites (Crinoidea): Bactrocrinites robustus n. sp. (Cantabrian Mountains, NW Spain). Revista Española de Paleontología, 23, 267-271.

Roemer, C. F. 1844. Das Rheinische Übergangsgebirge: Eine palaeontologisch-Geognostische Darstellung. Hahn, Hannover, 96 pp.

Schnur, J. 1849. Crinoiden. In: Die Versteinerungen des Übergangsgebirges der Eifel. Jahresbericht über den Schul-Cursus 1848/1849 an dem Gymnasium zu Trier (ed. J. Steininger). Published by the gymnasium, Trier, 21-22.

Schultze, L., 1866. Monographie der Echinodermen des Eifler Kalkes. Carl Gerold's Sohn, Wien, 1-118.

Truyols, J. \& Julivert, M. 1976. La sucesión paleozoica entre Cabo Peñas y Antromero (Cordillera Cantábrica). Trabajos de Geología, Universidad de Oviedo, 8, 5-30.

Manuscrito recibido: 31 de marzo, 2010 Manuscrito aceptado: 22 de noviembre, 2010 